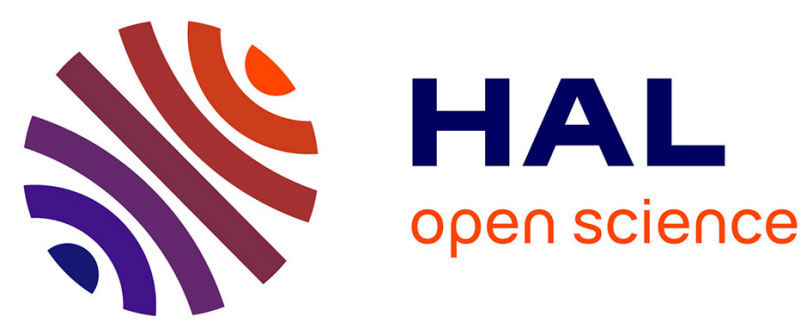

\title{
Activity of fulvestrant versus exemestane in advanced breast cancer patients with or without visceral metastases: data from the EFECT trial
}

Louis Mauriac, Gilles Romieu, José Bines

\section{- To cite this version:}

Louis Mauriac, Gilles Romieu, José Bines. Activity of fulvestrant versus exemestane in advanced breast cancer patients with or without visceral metastases: data from the EFECT trial. Breast Cancer Research and Treatment, 2008, 117 (1), pp.69-75. 10.1007/s10549-008-0141-z . hal-00535314

\author{
HAL Id: hal-00535314 \\ https://hal.science/hal-00535314
}

Submitted on 11 Nov 2010

HAL is a multi-disciplinary open access archive for the deposit and dissemination of scientific research documents, whether they are published or not. The documents may come from teaching and research institutions in France or abroad, or from public or private research centers.
L'archive ouverte pluridisciplinaire HAL, est destinée au dépôt et à la diffusion de documents scientifiques de niveau recherche, publiés ou non, émanant des établissements d'enseignement et de recherche français ou étrangers, des laboratoires publics ou privés. 


\title{
Activity of fulvestrant versus exemestane in advanced breast cancer patients with or without visceral metastases: data from the EFECT trial
}

\author{
Louis Mauriac · Gilles Romieu · José Bines
}

Received: 8 May 2008/Accepted: 15 July 2008/Published online: 23 November 2008

(C) Springer Science+Business Media, LLC. 2008

\begin{abstract}
Purpose Patients with visceral metastases (VM: lung and/or liver metastases) are generally regarded as being less responsive to hormonal therapy, and chemotherapy often becomes the default treatment. This paper reports a subgroup analysis from EFECT (The Evaluation of Faslodex versus Exemestane Clinical Trial) examining the efficacy of fulvestrant and exemestane in patients with or without VM. Methods EFECT is a randomised, doubleblind, multicentre, Phase III trial in postmenopausal women with advanced breast cancer progressing or recurring after prior non-steroidal aromatase inhibitor therapy. Results Overall, approximately $57 \%$ of patients in EFECT had visceral involvement. Fulvestrant and exemestane demonstrated clinical benefit in $29.1 \%$ and $27.2 \%$ of patients with VM, respectively. Median duration of response was 13.5 vs 10.8 months and median duration of clinical benefit was 9.9 vs 8.1 months, respectively. Conclusions These results encourage the use of endocrine agents such as fulvestrant in treating patients with advanced breast cancer and VM.
\end{abstract}

Keywords Advanced breast cancer .

Visceral metastases · Fulvestrant · Exemestane ·

Aromatase inhibitors $\cdot$ Clinical trial

L. Mauriac $(\bowtie)$

Institut Bergonié, Centre Régional de Lutte Contre le Cancer

de Bordeaux et du Sud-Ouest, 229 Cours de l'Argonne,

33076 Bordeaux, France

e-mail: mauriac@bergonie.org

G. Romieu

Centre Val D'Aurelle-Paul Lamarque, Montpellier, France

J. Bines

Instituto Nacional de Câncer, Rio de Janeiro, Brazil

\section{Introduction}

Advanced breast cancer patients with visceral metastases (VM) are often regarded as having a worse prognosis and being less likely to respond to hormonal therapy than those with metastases in soft tissue and bone [1]. To date, cytotoxic therapies have provided the mainstay of treatment for patients with VM, with various mono- and polychemotherapy regimens proving efficacious in such patients $[2,3]$. However, endocrine therapy, which generally has a better side-effect profile than chemotherapy, may also be an option in patients with hormone-sensitive disease.

Phase III clinical trials of endocrine treatments for patients with VM have been mainly limited to patients with visceral disease that is not rapidly progressing or symptomatic [4-6]. Analyses of treatment benefit in this subgroup of patients are important, as they provide insights into the usefulness of endocrine therapy as an alternative to chemotherapy in this clinical setting. To date there have been reports supporting the activity of both the oestrogenreceptor antagonist fulvestrant $\left(\right.$ Faslodex $\left.^{\mathrm{TM}}\right)$ and the steroidal aromatase inhibitor (AI) exemestane (Aromasin ${ }^{\mathrm{TM}}$ ) in patients with VM, but none comparing the activity of these agents in this setting.

Trials 0020 and 0021 were Phase III, second-line trials comparing the efficacy and tolerability of the non-steroidal AI anastrozole (Arimidex ${ }^{\mathrm{TM}}$ ) with fulvestrant, in the postanti-oestrogen setting. In a subgroup analysis of combined data from these trials, both agents demonstrated similar activity in patients with $\mathrm{VM}$, with objective response and clinical benefit (CB) endpoints favouring fulvestrant, although the differences between treatments were not statistically significant [6]. In another double-blind, randomised Phase III trial comparing exemestane versus 
megestrol acetate $\left(\right.$ Megace $^{\mathrm{TM}}$ ) after tamoxifen failure, exemestane demonstrated better activity than megestrol acetate in patients with VM [4].

Non-steroidal AIs are being increasingly used as the preferred first-line therapy in adjuvant and advanced disease settings (in place of tamoxifen). Unfortunately, treatment options are limited when patients experience either progression or recurrence on non-steroidal AIs. There have been limited data to support the activity of both fulvestrant and exemestane in this setting, suggesting a lack of cross-resistance between these treatments and the nonsteroidal AIs [7-11]. Thus, there is an increasing need for alternative and effective treatments that can be used after non-steroidal AI failure, particularly in patients with VM, who are generally considered difficult to treat. The Evaluation of Faslodex versus Exemestane Clinical Trial (EFECT) is a randomised, double-blind, multicentre, Phase III study comparing the efficacy and tolerability of fulvestrant versus exemestane in postmenopausal women with hormone receptor-positive advanced breast cancer who progressed or recurred after prior therapy with a non-steroidal AI (anastrozole or letrozole [Femara $\left.{ }^{\mathrm{TM}}\right]$ ) [12]. EFECT, therefore, assesses any cross-resistance between non-steroidal and steroidal AIs, as well as any crossresistance between AIs and fulvestrant. The current paper reports a subgroup analysis from EFECT, evaluating the efficacy of fulvestrant and exemestane in the subgroups of patients with or without VM.

\section{Methods}

\section{Patients}

Details of the study design and methodology of EFECT have been reported previously [12]. Briefly, EFECT (study code 9238IL/0048) is a randomised, double-blind, doubledummy, multicentre, Phase III trial comparing the efficacy and tolerability of fulvestrant versus exemestane in postmenopausal women with hormone receptor-positive, locally advanced or metastatic breast cancer. Eligible patients were those whose disease had progressed during treatment with a non-steroidal AI, or patients whose disease had recurred within 6 months of AI discontinuation. Patients could have received tamoxifen prior to non-steroidal AI therapy. Other eligibility criteria included: oestrogen receptor-positive (ER-positive) and/or progesterone-receptor positive (PgR-positive) disease, Eastern Cooperative Oncology Group performance status 0-2, life expectancy of $\geq 3$ months, and at least one measurable or evaluable lesion. Exclusion criteria included: the presence of life-threatening metastatic visceral disease, brain or leptomeningeal metastases, prior exposure to fulvestrant or exemestane, extensive radiation or cytotoxic therapy within the past 4 weeks, a history of bleeding diathesis or a need for long-term anticoagulant therapy. For the current analysis, patients were classified into those with or without VM (ie the presence of breast cancer metastases in the lung and/ or liver).

Patients were recruited from Canada, the United States, Europe, South America and South Africa from August 2003 to November 2005. All patients provided written informed consent, and the trial was conducted in accordance with the Declaration of Helsinki and all relevant local ethical committees.

\section{Treatment}

Fulvestrant was administered as an IM injection in a loading-dose regimen: $500 \mathrm{mg}$ on Day 0, $250 \mathrm{mg}$ on Days 14 and 28 , and $250 \mathrm{mg}$ every $28 \pm 3$ days thereafter. Exemestane $(25 \mathrm{mg})$ was given orally once daily. All patients received corresponding placebo medication. Treatment continued until disease progression, death or any other event necessitating treatment withdrawal; patients were followed up until death [12].

\section{Tumour assessments}

Up to ten measurable target lesions (no more than five lesions per organ) were selected at screening. These target lesions were monitored by the investigator throughout the study, with tumour dimensions recorded for each patient during each visit. All other (non-target) lesions were also monitored, using the same technique. Response and progression were calculated in comparison with baseline measurements. A patient was determined to have progressed if they showed progression of target lesions (for example, at least $20 \%$ increase in diameter), progression of existing non-target lesions, or the appearance of one or more new lesions. Although a central review of these assessments was not carried out, the Response Evaluation Criteria in Solid Tumours (RECIST) reports for each patient were validated centrally using a stringent algorithm.

Patients were examined by a physician every month until Month 6, and every 3 months thereafter. Tumours were assessed every 8 weeks from randomisation until Month 6, and then every 3 months thereafter, until disease progression or death from any cause.

\section{Study endpoints}

The primary endpoint of the overall study was time to progression, which was defined as the number of days from randomisation until objective disease progression (according to the RECIST) [13] or death from any cause. 
Secondary endpoints included objective response rate, $\mathrm{CB}$ rate and duration of response. The objective response rate was defined as the proportion of patients who had a best overall response of either a complete response (CR) or a partial response (PR), according to RECIST criteria. The $\mathrm{CB}$ rate was defined as the proportion of patients with a best overall response of CR, PR or stable disease for at least 24 weeks. The duration of response was calculated for patients who had a best overall response of $\mathrm{CR}$ or $\mathrm{PR}$, and was defined as the date from randomisation until the date of disease progression or death from any cause. The duration of $\mathrm{CB}$ was also retrospectively calculated for both the fulvestrant and exemestane treatment groups.

\section{Statistical analyses}

The primary and secondary endpoints were assessed in two prospectively defined subgroups, comprising patients with or without VM. Time to progression was summarised using Kaplan-Meier curves and Kaplan-Meier estimates of median times to event for each treatment group. Treatment comparisons were performed by a log-rank test (unadjusted model with treatment factor only). Results are presented as the hazard ratio (HR) of fulvestrant to exemestane with the corresponding $95 \%$ confidence intervals (CI) and $P$-values, where appropriate.

Objective response and $\mathrm{CB}$ were analysed using an unadjusted logistic regression model with treatment factor only. Results are presented as the odds ratio together with the corresponding $95 \% \mathrm{CIs}$ and $P$-values. The duration of response and duration of $\mathrm{CB}$ were also summarised using Kaplan-Meier curves and Kaplan-Meier estimates of median times to event for each treatment group. All measures were assessed on the intent-to-treat population.

\section{Results}

Patients

Overall, 693 women recruited into the EFECT trial were randomised to fulvestrant $(n=351)$ or exemestane $(n=342)$. Of these, $197(56.1 \%)$ patients receiving fulvestrant and 198 (57.9\%) patients receiving exemestane had visceral involvement. Both treatment groups were well balanced in terms of baseline demographics (Table 1). Overall, approximately $80 \%$ of patients in each group had received prior therapy with a selective oestrogen-receptor modulator (SERM; eg tamoxifen, raloxifene, tomerifene or LY353381). More than half of the patients (fulvestrant $57.1 \%$, exemestane $53.4 \%$ ) had received adjuvant therapy with a SERM, and approximately one-quarter (fulvestrant
$23.7 \%$, exemestane $26.4 \%$ ) had received a SERM in the advanced breast cancer setting. Less than $3 \%$ of patients in each group had received a SERM in both the adjuvant and advanced settings prior to receiving non-steroidal AI treatment.

\section{Time to progression}

The overall profile of the Kaplan-Meier curves suggested that time to progression was slightly shorter in patients with VM, compared with those without VM, irrespective of the treatment received (Fig. 1a, b). In patients with VM, the median time to progression was similar for fulvestrant compared with exemestane (3.1 vs 2.8 months, respectively; HR 0.92, 95\% CI 0.74, 1.13, $P=0.409$ ) (Fig. 2a). The median time to progression was slightly longer in patients without VM (fulvestrant 4.1 months, exemestane 5.2 months; HR $1.03,95 \%$ CI $0.80,1.33, P=0.817$ ) (Fig. 2a).

\section{Best overall response}

Overall, 270 patients (76.9\%) in the fulvestrant group and 270 patients $(78.9 \%)$ in the exemestane group were evaluable for response. The proportion of patients treated with fulvestrant who achieved an objective response was similar, regardless of visceral involvement $(7.1 \%$ of patients with $\mathrm{VM}$ and $8.0 \%$ of patients without VM) (Table 2). Patients with VM who were treated with exemestane had a lower objective response rate $(4.4 \%)$ compared with patients without VM (11.6\%), although the difference was not statistically significant (Table 2).

Both fulvestrant and exemestane demonstrated CB in patients with VM after AI failure. The number of patients experiencing $\mathrm{CB}$ or achieving stable disease for $\geq 24$ weeks was similar for both treatment groups (Table 2). Overall, the proportion of patients achieving CB or stable disease for $\geq 24$ weeks was lower in patients with VM than those without VM, irrespective of the treatment received.

\section{Duration of response and $\mathrm{CB}$}

In patients with VM, the median duration of response was longer with fulvestrant (13.5 months) compared with exemestane (10.8 months). The median duration of response was also longer with fulvestrant than with exemestane (11.7 vs 8.3 months, respectively) in patients without VM (Fig. 2b).

Similarly, the median duration of CB was slightly longer with fulvestrant (9.9 months) compared with exemestane (8.1 months) in patients with VM, whereas in patients without $\mathrm{VM}$, the median duration of $\mathrm{CB}$ was similar 
Table 1 Baseline patient characteristics and demographics

\begin{tabular}{|c|c|c|c|c|}
\hline & \multicolumn{2}{|c|}{ Fulvestrant $(n=351)$} & \multicolumn{2}{|c|}{ Exemestane $(n=342)$} \\
\hline & $\mathrm{VM}(n=197)$ & No VM $(n=154)$ & $\mathrm{VM}(n=198)$ & No VM $(n=144)$ \\
\hline Median age, years (range) & $63(38-88)$ & $63(40-86)$ & $63(32-91)$ & $63(37-85)$ \\
\hline \multicolumn{5}{|l|}{ WHO performance status, $n(\%)$} \\
\hline Normal activity $(0)$ & $109(55.3)$ & $85(55.2)$ & $104(52.5)$ & $77(53.5)$ \\
\hline Restricted activity (1) & $74(37.6)$ & $59(38.3)$ & $88(44.4)$ & $61(42.4)$ \\
\hline In bed $\leq 50 \%$ of the time (2) & $14(7.1)$ & $10(6.5)$ & $6(3.0)$ & $6(4.2)$ \\
\hline \multicolumn{5}{|l|}{ Prior treatments, $n(\%)$} \\
\hline Adjuvant endocrine therapy & $129(65.5)$ & $88(57.1)$ & $113(57.1)$ & $86(59.7)$ \\
\hline Endocrine therapy for advanced disease & $175(88.8)$ & $138(89.6)$ & $176(88.9)$ & $118(81.9)$ \\
\hline 1 prior endocrine therapy & $80(40.6)$ & $65(42.2)$ & $92(46.5)$ & $55(38.2)$ \\
\hline$>1$ prior endocrine therapy & $117(59.4)$ & $89(57.8)$ & $106(53.5)$ & $89(61.8)$ \\
\hline Adjuvant chemotherapy & $90(45.7)$ & $57(37.0)$ & $97(49.0)$ & $71(49.3)$ \\
\hline Chemotherapy for advanced disease & $55(27.9)$ & $32(20.8)$ & $46(23.2)$ & $28(19.4)$ \\
\hline Adjuvant radiotherapy & $111(56.3)$ & $79(51.3)$ & $104(52.5)$ & $67(46.5)$ \\
\hline Radiotherapy for advanced disease & $63(32.0)$ & $66(42.9)$ & $73(36.9)$ & $69(47.9)$ \\
\hline \multicolumn{5}{|l|}{ AI sensitivity, $n(\%)$} \\
\hline AI-sensitive disease & $132(67.0)$ & $92(59.7)$ & $124(62.6)$ & $86(59.7)$ \\
\hline AI-resistant disease & $65(33.0)$ & $62(40.3)$ & $74(37.4)$ & $58(40.3)$ \\
\hline \multicolumn{5}{|l|}{ Hormone-receptor status, $n(\%)$} \\
\hline ER-positive and PgR-positive & $136(69.0)$ & $101(65.6)$ & $112(56.6)$ & $81(56.3)$ \\
\hline Not ER-positive and PgR-positive & $61(31.0)$ & $53(34.4)$ & $86(43.4)$ & $63(43.8)$ \\
\hline \multicolumn{5}{|l|}{ Measurable disease } \\
\hline Yes & $182(92.4)$ & $88(57.1)$ & $184(92.9)$ & $86(59.7)$ \\
\hline No & 15 (7.6) & $66(42.9)$ & $14(7.1)$ & $58(40.3)$ \\
\hline
\end{tabular}

AI, aromatase inhibitor; ER, oestrogen receptor; PgR, progesterone receptor; VM, visceral metastases; WHO, World Health Organization

between treatments (8.0 and 8.6 months, respectively) (Fig. 2c).

Kaplan-Meier curves illustrating the duration of $\mathrm{CB}$ in patients with or without VM are presented in Fig. 3a, b, respectively.

\section{Discussion}

Treatment options for patients with advanced disease, particularly those with VM, remain ill defined. Such patients are considered to have a poorer prognosis and current treatment guidelines often recommend cytotoxic therapy as the treatment of choice [2]. However, there is a need for alternative treatments with more favourable toxicity profiles, particularly after progression or recurrence on initial hormonal therapy.

As the treatment goal for all patients with advanced disease is to prolong survival and maintain/improve quality of life, endocrine therapy is often the treatment of choice for patients with hormone receptor-positive disease that is not rapidly progressing. Recently, the overall analysis from the EFECT trial demonstrated that fulvestrant and exemestane have comparable efficacy and tolerability in postmenopausal women with advanced breast cancer following non-steroidal AI failure [12].

In the current subgroup analysis from EFECT, both fulvestrant and exemestane provided CB in approximately $30 \%$ of patients with VM. Furthermore, duration of response (13.5 vs 10.8 months) and duration of CB (9.9 vs 8.1 months) numerically favoured fulvestrant. While these small differences in efficacy could be due to the slightly higher proportion of VM patients with tumours positive for both ER and PgR in the fulvestrant group (69.0\%), compared with patients in the exemestane group (56.6\%), similar differences were also noted in the overall analysis population, where a median duration of response of 13.5 months was reported with fulvestrant vs 9.8 months with exemestane, and median durations of $\mathrm{CB}$ were 9.3 vs 8.3 months, respectively [12]. Although these endpoints were comparatively longer in the fulvestrant group, this trial was not powered to detect differences in the activity of these agents in patients with or without VM. Therefore, further prospectively-designed analyses are required to 


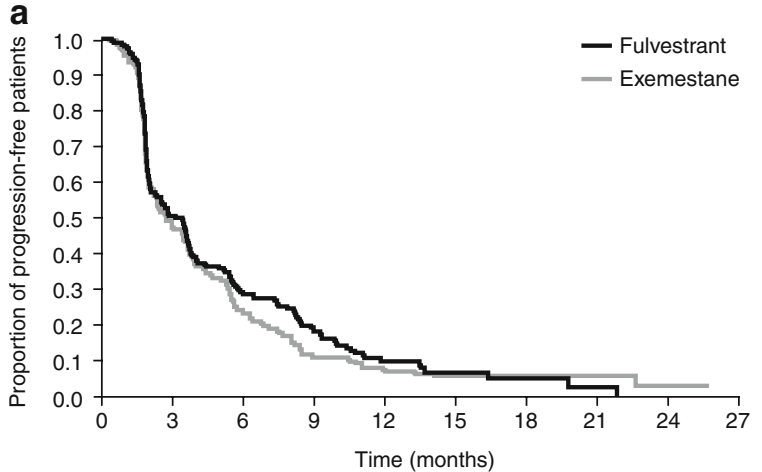

At risk:

$\begin{array}{llllllllll}\text { Fulvestrant } & 197 & 94 & 51 & 29 & 11 & 4 & 2 & 1 & 0 \\ \text { Exemestane } & 198 & 92 & 44 & 17 & 11 & 9 & 5 & 4 & 1\end{array}$

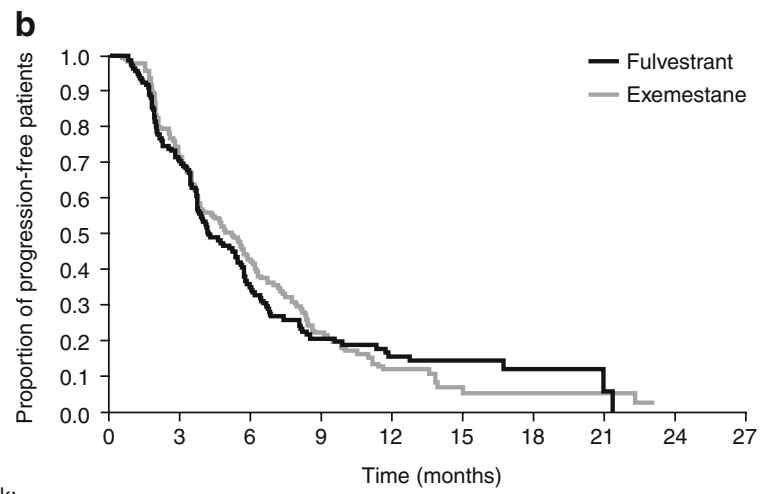

At risk:

$\begin{array}{llllllllll}\text { Fulvestrant } & 154 & 101 & 45 & 21 & 14 & 8 & 2 & 1 & 0\end{array}$

$\begin{array}{llllllllll}\text { Exemestane } & 144 & 98 & 54 & 24 & 10 & 3 & 3 & 2 & 0\end{array}$

Fig. 1 Kaplan-Meier curves for time to progression in patients (a) with visceral metastases or (b) without visceral metastases

ascertain whether fulvestrant offers any statistically significant benefits in patients with VM.

However, as seen previously [9-11] there was also evidence for a lack of cross-resistance between exemestane and the non-steroidal AIs; it has been speculated that this may be due to the androgenic effects of exemestane [12]. These data, therefore, suggest that both fulvestrant and exemestane may be considered as suitable options for patients with VM who are eligible for further endocrine therapy, following non-steroidal AI failure.

These findings confirm previous data on the activity of fulvestrant and exemestane in patients with or without VM following prior endocrine treatment. Combined data from two Phase III trials comparing fulvestrant and anastrozole following anti-oestrogen failure found that both drugs were active in patients with VM [6]. In line with the present analyses, some endpoints favoured fulvestrant, with objective response rates of $15.7 \%$ and $13.2 \%$ and median durations of response of 17.5 months and 11.7 months for fulvestrant and anastrozole, respectively [6]. Furthermore, pooled data from a compassionate-use programme of
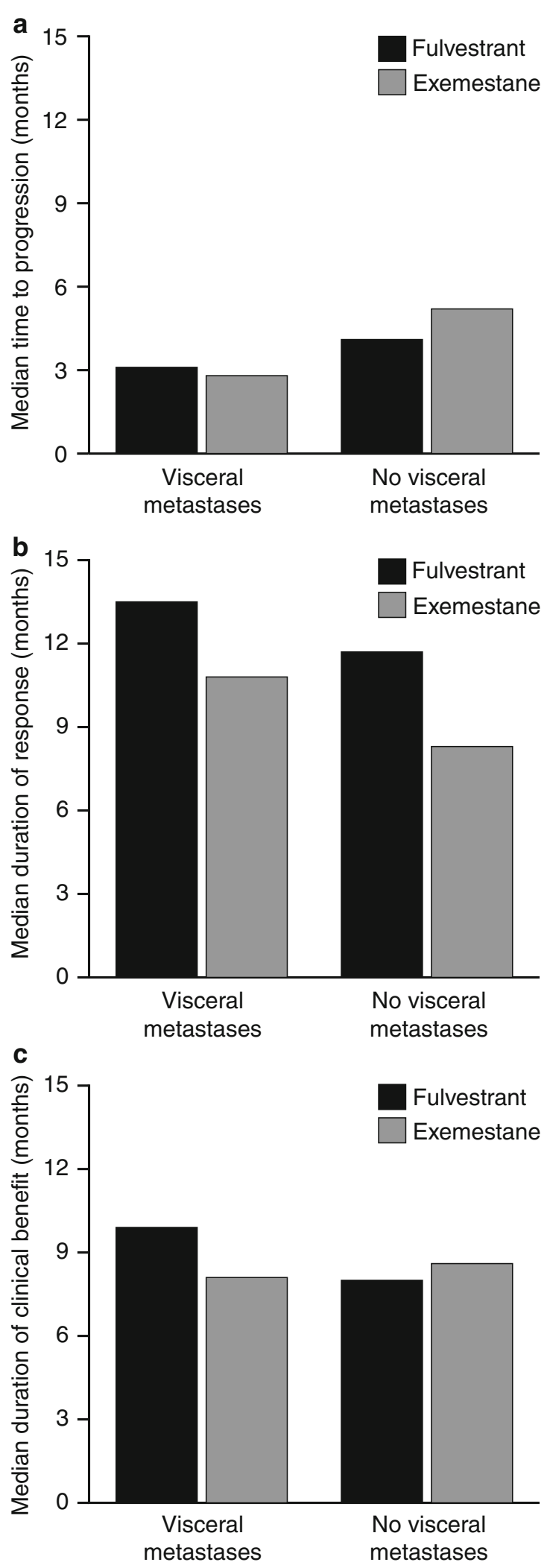

Fig. 2 Median time to progression (a), duration of response (b), and duration of clinical benefit (c) for patients with or without visceral metastases 
Table 2 Proportion of evaluable patients achieving an objective response (OR), clinical benefit (CB) or stable disease (SD) for $\geq 24$ weeks

\begin{tabular}{|c|c|c|c|c|}
\hline VM & Fulvestrant $(n=182)$ & Exemestane $(n=184)$ & Odds ratio $(95 \% \mathrm{CI})$ & $P$-value \\
\hline $\mathrm{OR}, n(\%)$ & $13(7.1)$ & $8(4.4)$ & $1.69(0.70,4.37)$ & 0.25 \\
\hline $\mathrm{CB}, n(\%)$ & $53(29.1)$ & $50(27.2)$ & $1.10(0.70,1.74)$ & 0.68 \\
\hline $\mathrm{SD} \geq 24$ weeks, $n(\%)$ & $40(22.0)$ & $42(22.8)$ & $\mathrm{NC}$ & $\mathrm{NC}$ \\
\hline No $\mathrm{VM}^{\mathrm{a}}$ & Fulvestrant $(n=88)$ & Exemestane $(n=86)$ & Odds ratio $(95 \% \mathrm{CI})$ & $P$-value \\
\hline $\mathrm{OR}, n(\%)$ & $7(8.0)$ & $10(11.6)$ & $0.66(0.23,1.80)$ & 0.41 \\
\hline $\mathrm{CB}, n(\%)$ & $34(38.6)$ & $35(40.7)$ & $0.92(0.50,1.69)$ & 0.78 \\
\hline $\mathrm{SD} \geq 24$ weeks, $n(\%)$ & $27(30.7)$ & $25(29.1)$ & $\mathrm{NC}$ & $\mathrm{NC}$ \\
\hline
\end{tabular}

${ }^{a}$ Only patients with measurable disease are included

CI, confidence interval; NC, not calculated; VM, visceral metastases

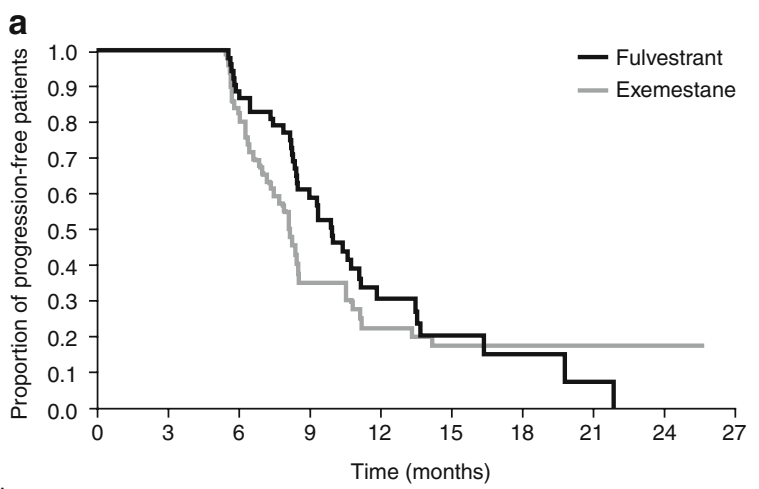

At risk:

$\begin{array}{llllllllll}\text { Fulvestrant } & 53 & 53 & 46 & 28 & 10 & 4 & 2 & 1 & 0\end{array}$

$\begin{array}{llllllllll}\text { Exemestane } & 50 & 50 & 40 & 14 & 9 & 7 & 3 & 3 & 1\end{array}$

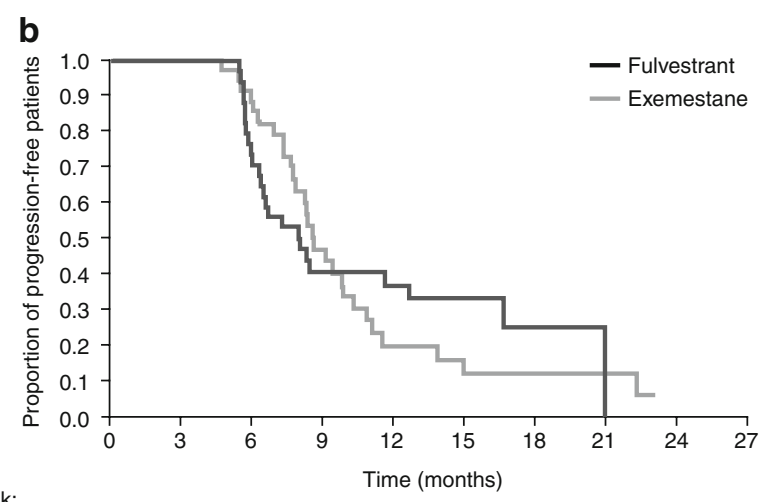

At risk:

$\begin{array}{llllllllll}\text { Fulvestrant } & 34 & 34 & 25 & 12 & 10 & 6 & 1 & 0 & 0\end{array}$

$\begin{array}{llllllllll}\text { Exemestane } & 35 & 35 & 29 & 14 & 5 & 3 & 3 & 2 & 0\end{array}$

Fig. 3 Kaplan-Meier curves for duration of clinical benefit in patients (a) with visceral metastases or (b) without visceral metastases

fulvestrant have reported CB in $32.4 \%$ of patients with VM [14]. Another compassionate-use study in Belgium has reported that $26.4 \%$ of patients with VM achieved CB following fulvestrant treatment [15]. With regard to exemestane, a previous Phase III trial evaluating this agent versus megestrol acetate found that $\mathrm{CB}$ rates were higher with exemestane $(36.3 \%)$ than with megestrol acetate
(30.0\%) in patients with VM, although the difference was not significant [4]. In addition, a recent case series where exemestane was given following several prior therapies, including non-steroidal AIs, described a CB rate of $33.0 \%$ for patients with visceral involvement [10].

Despite the available data, it is difficult to compare the activity of different endocrine treatments between trials, due to differences in baseline patient characteristics, line of treatment or endpoint definitions. Comparisons between the activity of endocrine therapy and chemotherapy in patients with VM are even more difficult, due to a lack of trials directly comparing such treatments. Nonetheless, our data add to the body of evidence suggesting that endocrine agents such as fulvestrant may be effective alternatives to chemotherapy in patients with visceral involvement in the appropriate setting, and that the presence of VM should not preclude the use of endocrine therapy.

Acknowledgements Dr Mauriac has acted as a consultant for, and received honoraria from, AstraZeneca and Novartis. Dr Romieu has no potential conflicts of interest. Dr Bines has acted as a consultant for, and received honoraria from, AstraZeneca. This study was sponsored by AstraZeneca. The sponsors were involved in the study design, and collection, analysis and interpretation of data. The authors would like to thank Sandra Cuscó, PhD, from Complete Medical Communications, who provided medical writing support funded by AstraZeneca.

\section{References}

1. Solomayer EF, Diel IJ, Meyberg GC, Gollan C, Bastert G (2000) Metastatic breast cancer: clinical course, prognosis and therapy related to the first site of metastasis. Breast Cancer Res Treat 59:271-278. doi:10.1023/A:1006308619659

2. National Cancer Institute PDQ Statement (2007) Breast cancer (PDQ): treatment. Stage IIIB, inoperable IIIC, IV, recurrent, and metastatic breast cancer. Available via http://www.nci.nih. gov/cancertopics/pdq/treatment/breast/HealthProfessional/page8. Accessed 8 May 2007

3. National Comprehensive Cancer Network (2007) NCCN Clinical practice guidelines in oncology, Version I: Breast cancer. Available via http://www.nccn.org. Accessed 30 Mar 2007 
4. Kaufmann M, Bajetta E, Dirix LY et al (2000) Exemestane is superior to megestrol acetate after tamoxifen failure in postmenopausal women with advanced breast cancer: results of a phase III randomized double-blind trial. The Exemestane Study Group. J Clin Oncol 18:1399-1411

5. Howell A, Robertson JF, Vergote I (2003) A review of the efficacy of anastrozole in postmenopausal women with advanced breast cancer with visceral metastases. Breast Cancer Res Treat 82:215-222. doi:10.1023/B:BREA.0000004375.17920.0b

6. Mauriac L, Pippen JE, Quaresma Albano J, Gertler SZ, Osborne CK (2003) Fulvestrant (Faslodex) versus anastrozole for the second-line treatment of advanced breast cancer in subgroups of postmenopausal women with visceral and non-visceral metastases: combined results from two multicentre trials. Eur J Cancer 39:1228-1233. doi:10.1016/S0959-8049(03)00199-0

7. Ingle JN, Suman VJ, Rowland KM et al (2006) Fulvestrant in women with advanced breast cancer after progression on prior aromatase inhibitor therapy: North Central Cancer Treatment Group Trial N0032. J Clin Oncol 24:1052-1056. doi:10.1200/ JCO.2005.04.1053

8. PereyL,ParidaensR,HawleHetal(2007)Clinicalbenefitoffulvestrant inpostmenopausalwomenwithadvancedbreastcancerandprimaryor acquired resistance to aromatase inhibitors: final results of phase II Swiss Group for Clinical Cancer Research Trial (SAKK 21/00). Ann Oncol 18:64-69.doi:10.1093/annonc/mdl341

9. Gennatas C, Michalaki V, Carvounis E et al (2006) Third-line hormonal treatment with exemestane in postmenopausal patients with advanced breast cancer progressing on letrozole or anastrozole. A phase II trial conducted by the Hellenic Group of Oncology (HELGO). Tumori 92:13-17

10. Steele N, Zekri J, Coleman R et al (2006) Exemestane in metastatic breast cancer: effective therapy after third-generation nonsteroidal aromatase inhibitor failure. Breast 15:430-436. doi: 10.1016/j.breast.2005.08.032

11. Carlini P, Michelotti A, Ferreti G et al (2007) Clinical evaluation of the use of exemestane as further hormonal therapy after nonsteroidal aromatase inhibitors in postmenopausal metastatic breast cancer patients. Cancer Invest 25:102-105. doi:10.1080/ 07357900701224789

12. Chia S, Gradishar W, Mauriac L et al (2008) A double-blind, randomized, placebo-controlled trial of fulvestrant versus exemestane following prior non-steroidal aromatase inhibitor therapy in post-menopausal women with hormone receptor-positive advanced breast cancer: results from EFECT. J Clin Oncol 26:1664-1670. doi:10.1200/JCO.2007.13.5822

13. Therasse P, Arbuck SG, Eisenhauer EA et al (2000) New guidelines to evaluate the response to treatment in solid tumors. J Natl Cancer Inst 3:205-216. doi:10.1093/jnci/92.3.205

14. Steger GG, Gips M, Simon SD et al (2005) Fulvestrant ('Faslodex'): clinical experience from the compassionate use programme. Cancer Treat Rev 31(Suppl 2):S10-S16. doi:10.1016/j.ctrv.2005. 08.009

15. Neven P, Paridaens R, Pelgrims G et al (2008) Fulvestrant ('Faslodex') in advanced breast cancer: clinical experience from a Belgian cooperative study. Breast Cancer Res Treat 109:59-65. doi:10.1007/s10549-007-9628-2 